\title{
Heterogeneity of uranium in water from south peninsular India
}

\author{
Reeba Maria Jose ${ }^{1}$, Sunil $\mathbf{A}^{1}$,Ben Byju $\mathbf{S}^{1}$, Christa E Pereira ${ }^{1}$, Vaidyan V K ${ }^{1}$ and Jojo P J $\mathbf{J}^{1,2^{*}}$ \\ ${ }^{1}$ Centre for Advanced Research in Physical Sciences, Department of Physics, \\ Fatima Mata National College, Kollam, 691001, Kerala, India \\ ${ }^{2}$ Department of Physics, University Malaya, Kuala Lumpur,50603, Malaysia \\ Email: jojo@um.edu.my ,jojo@fatimacollege.net
}

(Received Feb 2011; Published March 2011)

\begin{abstract}
The South Peninsular India is known to have very high levels of natural background radiation due to the monazite rich beach sand. Primordial uranium is ubiquitous and the heaviest radio toxic trace element available in all terrestrial substances at varying levels depending on the geology of the region. Uranium series supports several short-lived radioisotopes during its decay including radium. Uranium in drinking water is important in terms of the ingestion dose also. This report depicts the results of uranium analysis of 600 water samples collected from the south coast peninsular region extending parts of Kerala and Tamilnadu states of India through fission track registration technique. Results of the analyses of water samples collected from different sources in the south coast of India show that uranium concentrations vary from $0.82 \mu \mathrm{g} / \mathrm{l}$ to $7.32 \mu \mathrm{g} / \mathrm{l}$ equivalents to the specific activity of $10.33 \mathrm{~Bq} / \mathrm{m}^{3}$ and $92.23 \mathrm{~Bq} / \mathrm{m}^{3}$, respectively. Estimated daily intake of uranium through drinking water ranges from 20.22 to $18.58 \mathrm{mBq} / \mathrm{kg}$ and is lower than the recommended limits of intake. The heterogeneity in distribution of uranium in water bodies is due to the presence of monazite sand deposited in the coast over the years.
\end{abstract}

Key words: Uranium, HBRA, water, ingestion dose

https://doi.org/10.14331/ijfps.2011.330003

\section{INTRODUCTION}

The largest proportion of human exposure to radiation comes from natural sources - from external sources of radiation, including cosmic and terrestrial radiation, and from inhalation or ingestion of radioactive materials. The total annual effective dose to the human population from natural sources includes cosmic rays $(0.39 \mathrm{mSv})$ and terrestrial gamma rays $(0.46 \mathrm{mSv})$ along with considerable doses received by ingestion and inhalation of long-lived natural radio nuclides (WHO, 2005). Of all naturally occurring elements uranium is heaviest and radiotoxic with a very long half-life. It is a ubiquitous radioactive trace element found in almost all terrestrial substances in different levels of concentration. It also supports several short lived radioisotopes in its decay series including radium with potential radiological importance. Water plays an important role in the geophysical and geochemical processes, which slowly recycles the trace elements to and biosphere. Intake of higher levels uranium can lead its accumulation in the organs like kidney and can be carcinogenic (Singh et al 1998). Nephritis is the primary chemically induced effect of uranium on human health (Hursh and Spoor, 1973).

The Southern peninsular India especially the coastal region, specifically Chavara and Karunagappilly regions in Kerala and Manavalakkuruchi in Tamilnadu, are known to have very high levels of natural background radiation owing to the rare earths rich monazite sand present in large amount. The major radioactive sources responsible for exposure are naturally occurring nuclides in the earth's crust such as ${ }^{232} \mathrm{Th},{ }^{238} \mathrm{U},{ }^{40} \mathrm{~K}$ which occur in abundance in minerals such as monazites and zircons. These earth-born radio nuclides impart not only the external radiation dose to the human beings but also causes ingestion and inhalation doses to human body through intakes of air, water and food (Rawat et al, 1991). The monazite sand in these regions contain about $9 \%$ thorium oxide, $0.35 \%$ uranium oxide along with phosphorus pentoxide, rare earths, and oxides of titanium, cerium, iron and silicon (Chougaonkar et al, 2004). 
Naturally, the ingestion doses would comprise of the long-lived radio nuclides through the intake of water, milk, fish, livestock and the vegetables grown locally. Scientific evidences from both human and animal studies that radiation exposure at low to moderate doses may increase the long-term incidence of cancer. Animal studies in particular suggest that the rate of genetic malformations may be increased by radiation exposure. According to World Health Organization (WHO) no deleterious radiological health effects are expected from consumption of drinking-water if the concentrations of radio nuclides are below the guidance levels equivalent to a committed effective dose below $0.1 \mathrm{mSv} /$ year (WHO, 2005). A systematic study has been conducted to evaluate the levels of uranium in drinking water from the natural resources in the southern peninsular India, spreading over the states of Kerala and Tamilnadu. Effective doses resulting from the intake of natural radio nuclides can be determined directly from measuring its concentrations in the body or estimated from concentrations in intake materials such as air, food and water. The published data available in the literature shows that natural radioactivity in water varies over a large range, mainly depending on the geological characteristics of the soils, and some measured concentrations largely exceed the reference values accepted for drinking water.

\section{MATERIALS AND METHODS}

The region selected for the study was the coastal strip extending three district in Kerala namely Allappuzha, Kollam and Thiruvananthapuram and Kanyakumary district in Tamilnadu. The investigation first involves the selection of sampling sites and sampling procedure. The sampling locations were selected within one kilometer strip along the coastal peninsula including the High Background Radiation Area (HBRA). A total of 98 locations were selected and five to seven samples were collected from each location making a total of 600 samples. Samples were coded properly according to their sources. The study area is bound by latitude $8^{\circ} 00^{\prime} \mathrm{N}$ and $9^{\circ} 20^{\prime} \mathrm{N}$ and longitude $76^{\circ} 20^{\prime} \mathrm{E}$ to $76^{\circ} 80^{\prime} \mathrm{E}$ approximately.

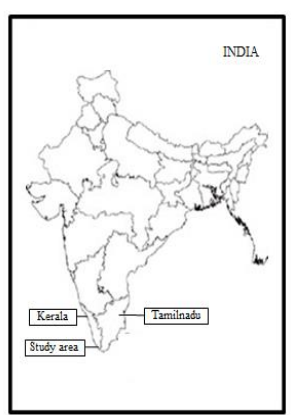

Figure 1. The experimental area

Samples were collected only from the natural water bodies namely pond, well and bore well, which were used for drinking. Sample collection was done in six phases covering the study area. Care was taken to have a geographically uniform distribution in choosing locations for sample collection. Small and clean plastic bottles ( $20 \mathrm{ml}$ capacity) were used for sample collection. Bottles were pre-rinsed with distilled water and then with the experimental water at the time of sample collection. Each sample brought to the laboratory was acidified by adding a drop of nitric acid in order to minimize the loss of uranium through absorption in the bottles. Water samples were collected mostly from the surface of the water bodies in the case of pond and well. Altogether 600 samples were collected in six phases from various sources for analysis.

Fission track registration technique is a very sensitive and reliable method for analyzing uranium. This method is capable of determining uranium levels even in sub-ppb (particles per billion) levels and is relatively cheaper also. Experimental technique known as 'dry method' has been used for the analyses (Jojo et al, 1994).Dielectric fission track detectors (Makrofol $\mathrm{KG}$ ) cut in the form of circular discs of $1.3 \mathrm{~cm}$ diameter were properly washed and rinsed with the double distilled water. They were carefully numbered and were arranged on a tray. Each drop of water samples $(0.05 \mathrm{ml})$ was placed on each disc using a micropipette.

Then, they were allowed to evaporate in a hot air oven at about $60^{\circ} \mathrm{C}$ to leave a circular residue of non-volatile substances in the water samples including natural uranium. Each detector disc with the non-volatile residue, including uranium, is then covered with another identical detector disc. The pair of discs is then sealed with polypropylene tapes to form a pellet of the sample (Bansal et al, 1992). These pellets were encapsulated in aluminum can of about $5 \mathrm{~cm}$ length and $1.5 \mathrm{~cm}$ diameter. A blank pellet, without any water sample residue in it, was also placed in the can to assess the background tracks, if any. A pair of circular pieces of micro-slides, which acts as neutron flux dosimeters, was also kept in the can. This capsule was sent for irradiation at Bhabha Atomic Research Centre (BARC), Bombay in the thermal column of the APSARA Reactor at a flux of $10^{16} \mathrm{nvt}$ for $3 \mathrm{~h}$. After receiving the irradiated samples, from BARC the detector discs were separated and washed thoroughly with water. Then they were etched in $6.25 \mathrm{~N} \mathrm{KOH}$ solution at $60^{\circ}$ for 20 minutes so that the fission tracks were developed into clearly visible size (Jojo et al, 1994).

The tracks were seen in a circular region where the evaporated water drop had left a residue of non-volatile matter including uranium in the water sample. The distribution of tracks was such that the circular region had a non-uniform distribution of tracks. The rim of the circular region had a higher track density and the interior portion had almost a uniform distribution of tracks. This is due to the fact that the water drop leaves maximum nonvolatile residue along the rim during evaporation. To find the total number of tracks on the detector, the rim as well as the interior was scanned separately using an optical transmission type research microscope at a magnification of 400X.Using optical scanning total number of tracks was determined on the detector discs following the standard protocol (Jojo et al, 1994, Fleicher and Delany, 1976).

Deducting the background tracks recorded in the blank pellet, the actual number of tracks was found for both the detector discs of 
each pellet. The average was taken as the total number $(\mathrm{N})$ of tracks for the sample. To obtain the thermal neutron dose, the irradiated standard glass placed in the capsule was cut to form a fresh surface. The glass piece was etched in $48 \%$ Hydrogen Fluoride at $23^{\circ} \mathrm{C}$ for 5 seconds to make the tracks visible and is scanned to obtain the fission track density. The neutron dose was calculated using the relation (Bansal et al, 1992):

$$
\phi=K \rho
$$

Where $\mathrm{K}$ is a constant $\left(=1.028 \times 10^{11}\right)$ depending on the material of the standard glass used as flux dosimeter and $\rho$ is the fission track density in the standard glass. With these known factors, uranium concentrations in water samples were calculated using the equation (Jojo et al, 1994):

$$
C_{w}=\left[(N M) /\left(V G N_{a} E \sigma \phi I\right)\right]
$$

Where $\mathrm{C}_{\mathrm{w}}$ is the concentration of uranium in water samples, $\mathrm{N}$ the total number of tracks, $\mathrm{M}$ the atomic weight of the fissile material (235), $\mathrm{V}$ the volume of the water droplet, $\mathrm{N}_{\mathrm{a}}$ Avogadro's number, $\mathrm{E}$ the etching efficiency of the Makrofol-KG detector, $\sigma$ fission cross section of the fissile isotope (580 barns), $\phi$ the neutron flux used and I the isotopic abundance ratio (Jojo et al, 1994).

\section{RESULTS AND DISCUSSION}

Results of the analyses of water samples collected from different sources in the south coast of India show that uranium concentrations vary from $0.82 \mu \mathrm{g} / \mathrm{l}$ to $7.32 \mu \mathrm{g} / \mathrm{l}$ equivalents to the specific activity of $10.33 \mathrm{~Bq} / \mathrm{m}^{3}$ and $92.23 \mathrm{~Bq} / \mathrm{m}^{3}$, respectively. The arithmetic mean (AM), geometric mean (GM) and geometric standard deviation (GSD) of the measured values for the two zones are presented in the Table 1.

In general there is a clear elevation in the uranium concentration in the well and pond water samples collected from the state of Kerala as compared with those from Tamilnadu as indicated by the AM and GM values.

This can be attributed to the abundance of monazite sand available in plenty in the coastal region of Kearla. It is reasonable to assume that uranium is transferred to local water bodies through leaching. The skewness and Kurtosis of the measured data are also shown in the table 1.

In Kerala, the concentration of uranium for well water is slightly positively skewed meaning that majority of the data are slightly lower than the average value. The data for pond showed a highly left (negatively) skewed distribution with most the values concentrated on the right of the mean, with extreme values to the left. This indicates that the concentrations of uranium in these samples are generally higher than the mean value presented in the table 1. For bore well the data is somewhat symmetric about the mean.

For Tamilnadu, both well and pond water showed a highly right skewed distribution indicating the exuberance of values lower than the mean. In this case also the bore water showed an approximately symmetric distribution about the mean. For Kerala, uranium concentrations in well water showed a leptokurtic distribution with a well-defined central peak.

The pond water samples showed a platykurtic curve with a broad centre. The mesokurtic distribution of bore well indicated a normal distribution. For Tamilnadu, water samples from well, pond and bore well showed platykurtic distribution with different degrees of kurtosis.

Table.1 Measured mean values of uranium concentration in the two states.

\begin{tabular}{c|ccc|ccc}
\hline & \multicolumn{3}{|c|}{ Kerala } & \multicolumn{3}{c}{ Tamilnadu } \\
\hline Source & Well & Pond & Bore well & Well & Pond & Bore well \\
Sample size & 186 & 118 & 148 & 63 & 46 & 39 \\
AM $(\mu \mathrm{g} / 1)$ & 4.24 & 3.26 & 1.44 & 1.18 & 1.12 & 1.98 \\
GM $(\mu \mathrm{g} / 1)$ & 4.02 & 3.28 & 1.28 & 0.98 & 1.02 & 0.96 \\
GSD & 2.68 & 2.14 & 2.11 & 1.08 & 1.22 & 1.12 \\
Skewness & 0.48 & -1.66 & 0.14 & 1.27 & 1.48 & 0.26 \\
Kurtosis & 2.62 & 0.86 & 2.48 & 2.22 & 3.85 & 2.43 \\
\hline
\end{tabular}

The obtained data was analyzed statistically to find any correlation between the levels of uranium in various sources using Pearson's method (Garrett,1981). The analysis was done between the average values of uranium concentrations from the three types of sources for the fifty eight locations.

It showed good positive correlation $(r=0.765$ at 0.05 level of significance) between the levels of uranium in pond and well for Kerala.

No such correlation could be obtained among the samples for Tamilnadu. Estimated daily intake of uranium through drinking water for both the regions ranges from 20.22 to $18.58 \mathrm{mBq} / \mathrm{kg}$ and is lower than the recommended limits of intake. WHO suggests a guidance value of Tolerable Daily Intake (TDI) of 15 $\mu \mathrm{g} / \mathrm{l}$ equivalent to $370 \mathrm{mBq} / \mathrm{kg}$ assuming a $60 \mathrm{~kg}$ adult consuming 21 water per day (WHO, 2005).

The concentrations of uranium measured in the present work shows that they are well within the suggested limit. A recent study conducted in the packaged drinking water samples in India found that the range of uranium in water between $0.04 \mu \mathrm{g} / \mathrm{l}$ to $3.88 \mu \mathrm{g} / \mathrm{l}$ (Sahoo et al, 2010).

\section{CONCLUSIONS}

From the present observations it can be concluded that the concentration of uranium level vary considerably from natural source to source and place to place. There is no alarmingly high uranium concentration in any of the samples analyzed. The measured values are in good agreement with the reported values in the contemporary literature.

The distribution of uranium in water bodies shows a heterogeneous nature of distribution. It is observed that the water bodies near to the sea have higher levels of uranium concentration. There is a positive correlation between the uranium levels in water collected from the pond and well samples along beach area. The daily intake of uranium through drinking water in the region is much less than the tolerable intake limit. 


\section{ACKNOWLEDGMENT}

The corresponding author (PJJ) thankfully acknowledges the financial assistance extended by
Science Technology and Environment Committee (STEC), Government of Kerala, India to conduct the study as a Research Project.

\section{REFRENCES}

Bansal, V., Rawat, A., Jojo, P.J., Prasad, R., 1992.Anlysing uranium concentration in drinking water samples in India using the fission track technique. Health phys. 62,3: 257-260.

Chougoanakar, M.P., Eappen, K.P., Ramachandran, T.V., Shetty, P.G., Mayya, Y.S., Sadasivan, S., Veknat, Raj, V., 2004. Profiles of doses to the population living in the high background radiation areas in Kerala, India. J. Env. Rad. 71: 275- 297

Fleicher, R.L., Delany, A.C., 1976. General Electric Corporation (GEC) Report No.76 CRDO 11: 124

Garrett, H.E., 1981. Statistics in Psychology and Education.Vakils, Feffer and Simons Ltd., Bombay 6: 143.

Hursh, J.B.,Spoor, N.L., 1973. Handbook of Experimental Pharmacology. Springer-Verlag, Berlin 36: $197-240$

Jojo, P.J., Rawat, A., Kumar, A., Prasad, R, 1994. Trace uranium analysis of water from the south west coastal region of India. J. Radioanal. Nucl. Chem. 178: 245-251.

Rawat, A., Jojo, P.J., Khan, A.J., Tyagi, R.K., Prasad, R., 1991.Radon exhalation rate in buildingmaterials. Nucl.Tracks and Radiat. Meas. 19, 1-4: 391

Sahoo, S.K., Mohapatra. S., Chakrabarty, A., Sumesh, C.G., Jha, V.N., Tripathi, R.M., Puranik, V.D., 2010. Determination of uranium at ultra-trace level in packaged drinking water by laser fluorimeter and consequent ingestion dose. Radioprotection 45: $55-66$.

Singh, A.K., Kumar, A., Jojo, .PJ., Prasad, R., 1998. Microanalysis of uranium in Indian soil samples using solid state nuclear track detection technique. $\mathrm{J}$ of Radio anal and NuclChem, 238,1-2:21-24

World Health Organisation (2005) Guidelines for drinking water quality, WHO/SDE/WSH/03.04/118, Geneva 